Discrete Comput Geom 29:477-481 (2003)

DOI: $10.1007 / \mathrm{s} 00454-002-2835-\mathrm{z}$

\title{
Covering the Plane with Fat Ellipses without Non-Crossing Assumption*
}

\author{
Aladár Heppes \\ Vércse u 24/A, 1124 Budapest, Hungary \\ hep9202@helka.iif.hu
}

\begin{abstract}
Kershner proved in 1939 that the density of a covering of the plane by congruent circles is at least $2 \pi / \sqrt{27}$ [3]. In 1950 L. Fejes Tóth [2] extended this result showing that the same density bound holds for coverings with congruent ellipses which do not "cross". In the present paper we prove that the non-crossing assumption is not necessary if the ellipses are sufficiently "fat".
\end{abstract}

\section{Introduction}

According to the classical theorem of Kershner [3] the density of a covering of the Euclidean plane with congruent circular discs cannot be smaller than $2 \pi / \sqrt{27}$, the density attained by the circumcircles of the meshes of a regular hexagonal mosaic. This result has been essentially extended by L. Fejes Tóth to convex centrally symmetric domains. He proved [2] that when a convex hexagon $H$ of the plane is covered by $k \geq 1$ non-crossing copies of a centrally symmetric convex domain $D$, then the covering density cannot be smaller than

$$
|D| / h(D),
$$

where $|D|$ and $h(D)$ denote the area of $D$ and the maximum of the area of a hexagon inscribed into $D$, respectively. (Two domains are said to cross, if for each of them the part of the domain not belonging to the other domain is not connected.) Clearly, the bound is exact for $k=1$ and it is sharp asymptotically, i.e. for the whole plane.

To get the best density bound for such a covering the non-crossing assumption cannot simply be dropped, in general. Let $H$ be a square covered by two hexagonal domains, each the convex hull of the midpoints of the four sides and the endpoints of a diagonal

* This research was supported by the Hungarian National Science Foundation OTKA, Grant No. T030012. 
of the square. Clearly, the square cannot be covered by these two domains unless they are crossing. This observation of the author has been generalized by Wegner [4], who proved that for any $k \geq 2$ a hexagonal domain $H$ and a convex domain $D$ can be found such that $H$ can be covered by $k$ copies of $D$, but only so that the copies cross in pairs. In spite of these results it seems very likely that for coverings of the whole plane Fejes Tóth's bound is valid without the "annoying" non-crossing assumption. L. Fejes Tóth himself suggested investigating the problem for domains which are close to the shape of a circle. Our approach is an attempt in this direction.

\section{The Theorem}

Let

$$
f=\frac{g_{7}-g_{6}}{g_{6}-g_{5}}=0.6275 \ldots,
$$

where $g_{k}=k \sin (2 \pi / k) / 2$ is the area of a regular $k$-gon inscribed into a unit circle. An ellipse is called fat if its half axes are 1 and $b$, where $b \geq b_{0}=0.8561 \ldots$, the value for which $b_{0}^{3}=f$.

In this paper we consider coverings of the plane by congruent fat ellipses and our goal is to prove the following:

Theorem. If a convex hexagon $H$ is covered by a finite number of congruent fat ellipses, then the covering density is at least $2 \pi / \sqrt{27}$.

Proof of the Theorem. The proof rests on two lemmas.

Lemma 1. Let $P$ be a point on the boundary of an ellipse of half axis 1 and $b<1$, let $l_{1}$ be a line touching the ellipse at $P$ and let $l_{2}$ be a line orthogonal to $l_{1}$ and containing the center of the ellipse. The maximum of the distance from $P$ to $l_{2}$ is $1-b$.

The proof of this lemma, presented in Section 3, is due to E. Molnár.

Lemma 2. The density of a covering of a convex hexagon $H$ with a finite number of non-crossing ellipses is at least $2 \pi / \sqrt{27}$ if the rate of the areas of any pair is $\geq f$.

This lemma is a special case of a result of K. Böröczky and G. Fejes Tóth (see [1]). First we show that the following holds:

Proposition. If $K_{i}$ and $K_{j}$ are crossing fat ellipses of small half axes $b_{i} \geq b_{0}$ and $b_{j} \geq b_{0}$, then the distance between their centers is at most

$$
d=\sqrt{5}\left(1-b_{0}\right) .
$$

Proof of the Proposition. To obtain an upper bound on the distance in question consider a pair of ellipses meeting in four distinct points. The union of the ellipses is decomposed 


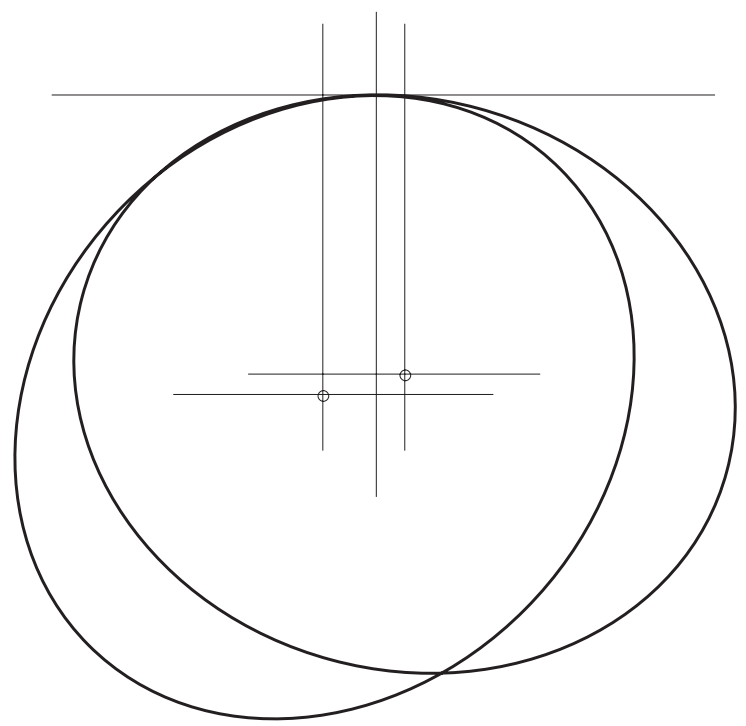

Fig. 1

by the curves into five parts: their intersection and four simply covered domains of disjoint interior. We now translate one of the ellipses so that the distance between their centers increases until they stop crossing. It is easy to see that during the translation the five domains mentioned above change continuously and in the limiting configuration one of the simply covered parts reduces to a single common point of the two boundaries, where the two tangent lines coincide.

Applying Lemma 1 to each of the ellipses and to the common tangent line as $l_{1}$ we obtain that the distance between the orthogonal projections of the centers on the common tangent is $\leq\left(1-b_{i}\right)+\left(1-b_{j}\right) \leq 2\left(1-b_{0}\right)$.

On the other hand, the difference of the distances from the two centers to this tangent line is at most $1-b_{0}$ and the claim of the proposition follows (Fig. 1).

Let us return to the proof of the theorem. Our proposition implies that a pair of crossing fat ellipses can be covered by a circle of radius $R$, if $R$ satisfies

$$
R \geq 1+d / 2=1,1608 \cdots
$$

Suppose that $K_{i}$ and $K_{j}$ is a crossing pair. Let us replace them by a disc of radius $R$ covering the union of both ellipses. As by each such replacement the number of the (original) crossing pairs is decreased by at least one, another covering can be constructed (in a finite number of steps) consisting of (original) non-crossing ellipses and discs of radius at least $1+d / 2$ to an arbitrary covering of $H$ with fat ellipses.

Clearly, neither the (original) ellipses of the new system nor the new discs of radius $R$ cross among themselves. Since the smallest curvature of a fat ellipse is $\geq b_{0}$ the ellipses and the discs do not cross either if

$$
R \geq 1 / b_{0}=1,1680 \cdots .
$$


(Should an ellipse and a circle cross, the circle could be moved continuously, maintaining the crossing property, so that finally the circle would touch the ellipse from the inside at the end of its shorter axis. This, however, contradicts our assumption on the curvatures.)

If (3) holds, condition (2) is fulfilled, so let $R=1 / b_{0}$. As $\left(1 / b_{0}\right)^{2}=1,3642 \cdots<$ $2 b_{0}=1,7122 \cdots$, the area of a replacing disc is smaller than the total area of the two replaced ellipses, thus by the replacements the density of the covering does not increase. Then, since the rate of the areas of the ellipses and the discs is just $f$ Lemma 2 can be applied for the new non-crossing system of ellipses to get the claim of the theorem.

\section{Proof of Lemma 1}

It can be assumed that $l_{1}$ is the $y$-axis and $P$ is the "top", the point of the largest $y$ coordinate of the ellipse $E(\varphi)$, which is obtained by applying (positive) rotation with angle $0 \leq \varphi \leq \pi / 2$ to the ellipse $E(0)$ defined by the equation

$$
(x)^{2}+(y / b)^{2}=1, \quad b<1 .
$$

Our purpose is to find the maximum of the $x$-coordinate of the top as a function of $\varphi$.

Using the notation

$$
\begin{aligned}
& x=\cos \alpha, \quad-\pi<\alpha \leq \pi, \\
& y=b \sin \alpha,
\end{aligned}
$$

the $(x, y)$-coordinates of the points of the rotated ellipse in the new system are

$$
\begin{aligned}
& X(\alpha, \varphi)=\cos \varphi \cos \alpha-b \sin \varphi \sin \alpha, \\
& Y(\alpha, \varphi)=\sin \varphi \cos \alpha+b \cos \varphi \sin \alpha
\end{aligned}
$$

and the top point (for a given rotation $\varphi$ ) is defined by the maximum of $Y(\alpha, \varphi)$ in $0 \leq \alpha \leq \pi / 2$.

Introducing the substitution

$$
\begin{aligned}
& \frac{\sin \varphi}{\sqrt{\sin ^{2} \varphi+b^{2} \cos ^{2} \varphi}}=\sin \psi, \\
& \frac{b \cos \varphi}{\sqrt{\sin ^{2} \varphi+b^{2} \cos ^{2} \varphi}}=\cos \psi
\end{aligned}
$$

yields

$$
Y(\alpha, \varphi)=Y(\alpha, \psi)=\sqrt{\sin ^{2} \varphi+b^{2} \cos ^{2} \varphi} \cdot \sin (\psi+\alpha) .
$$

Clearly, for any given $\varphi$ the top point is received for $\psi=\pi / 2-\varphi$, and its $x$-coordinate is

$$
X^{*}=\frac{1-b^{2}}{\sqrt{1 / \cos ^{2} \varphi+b^{2} / \sin ^{2} \varphi}}=\frac{1-b^{2}}{\sqrt{\tan ^{2} \varphi+1+b^{2} \cot ^{2} \varphi+b^{2}}} .
$$

$X^{*}$ is largest, when $\tan ^{2} \varphi+b^{2} \cot ^{2} \varphi$ is smallest possible. As the product of these two terms is constant $\left(b^{2}\right)$, their sum is minimal if they are equal, i.e. for $\tan \varphi=\sqrt{b}$. Then $X^{*}=1-b$ as claimed. 


\section{Remarks}

(1) It is an interesting question in its own right about what is a sharp estimate on the distance between the centers of crossing ellipses, since the estimate in (1) does not seem to be best possible.

(2) In the theorem, coverings with ellipses are considered. Clearly, similar results can be derived for domains which only approximate an ellipse. Not even the central symmetry of the domains seems to be essential.

(3) By stipulating stricter condition on fatness the requirement of equal area could be relaxed, as was done in [1] in case of non-crossing domains.

\section{References}

1. Fejes Tóth, G., Covering the plane by convex discs, Acta Math. Acad. Sci. Hungar., 23 (1973), 263-270.

2. Fejes Tóth, L., Some packing and covering theorems, Acta Sci. Math. Szeged, 12/A (1950), 62-67.

3. Kershner, R., The number of circles covering a set, Amer. J. Math., 61 (1939), 665-671.

4. Wegner, G., Zu einem ebenen Überdeckungsproblem, Studia Sci. Math. Hungar., 15 (1980), 287-297.

Received November 19, 2001, and in revised form August 6, 2002. Online publication January 30, 2003. 